\title{
Can Mobile Web Surveys Be Taken on Computers? A Discussion on a Multi-Device Survey Design
}

\author{
Marika de Bruijne ${ }^{1}$, Arnaud Wijnant ${ }^{1}$ \\ ${ }^{1}$ CentERdata, Tilburg University \\ Keywords: touchscreen, survey design, web survey, mobile web survey \\ https://doi.org/10.29115/SP-2013-0019
}

Survey Practice

Vol. 6, Issue 4, 2013

\begin{abstract}
This paper introduces an approach to designing a multi-device web survey by using a mobile touchscreen layout as base. We present the findings of an experiment in which we provided one half of an online panel with a survey designed for mobile browsers, and the other half with a standard web survey. Both questionnaire versions were accessible via regular as well as mobile browsers. Based on these results, we discuss whether it is feasible to use a survey with a mobile web layout as the main survey instrument for web respondents. For designing a multi-device, multi-browser survey, we suggest two strategies to be investigated further: optimizing the survey layout for each type of device separately, or optimizing a single design that is compatible with all devices.
\end{abstract}

\section{Background}

Led by increasing web usage on mobile devices, respondents are spontaneously accessing web questionnaires via their mobile device. In August 2013, about 11 percent of the respondents in the CentERpanel, an online probability panel in the Netherlands, reported to complete the panel's regular web surveys using tablets, and 2 percent using smartphones. These shares are expected to grow along with the increasing mobile internet penetration. If web surveys are not adapted for mobile browsers, taking a survey using a mobile device is likely to result in suboptimal questionnaire presentation and completion.

A few strategies for handling spontaneous mobile response have been suggested. Callegaro (2010) presents four approaches: blocking the unintended mobile respondents from taking part in the survey, flagging them in the data, developing a mobile questionnaire version for the most common mobile devices, or developing a survey platform that can handle all desktop and mobile devices.

Buskirk and Andrus (2012) refer to passive and active approaches to offering mobile web surveys. The passive way is to allow the respondent to access a web survey using mobile devices but not to modify the layout. The active approach is to reformat and deploy the survey for mobile browsers, applied when the survey is accessed via mobile devices. The latter approach is also referred to as responsive web survey design, which can be applied by detecting the respondent's browser type based on the automatically logged user agent string as soon as he or she accesses the questionnaire. Depending on the browser type, the respondent is either directed to a regular computer or a mobile version of the questionnaire (see e.g., Boreham and Wijnant 2013). 
Table 1 Main differences between computer web and mobile web layout.

Computer web layout

Designed for large screens, fixed font, and button size

Can include large amounts of information per screen

Requires accurate mouse handling

Various layout choices, e.g., grids and horizontal or vertical answer scales

Some features such as grids and horizontal scales do not fit small screens

Familiar to respondents, web standard
Mobile web layout

Designed for varying screen sizes (small to average), responsive font, and button size

A limited amount of information per screen

Relatively large buttons that are easy to select even with a finger

Question items usually on individual screens, vertical answer scales

Fits all screens

Less familiar layout, still in development

Computer and mobile versions of web surveys differ with regard to a few fundamental functional choices. Designed for computers, the regular web layout presumes large screens with mouse handling and typically has fixed parameters such as font and button sizes. Mobile web surveys, on the other hand, are designed for fingertip navigation on small to average screens and apply flexible font and button sizes which respond to the screen size. Further, implementation choices such as avoiding long horizontal answer scales (Stapleton 2013), presenting question items on individual screens, and limiting the question wording make a survey further suited to small screens. A comparison of the two layouts is presented in Table 1.

When actively designing a multi-device online survey, one commonly takes the standard computer-web design as the starting point and adapts it to mobile devices. Creating two separate versions of a questionnaire may, however, require considerably more development and testing time. Different layouts might also affect the response behavior, although comparisons between computer and mobile web surveys have shown very few differences in survey results or data quality (de Bruijne and Wijnant 2013; Mavletova 2013). Lower response rates and longer completion times in mobile web surveys have been reported, but these differences may also be due to device difference rather than layout.

There is another approach to offering a web survey via both computer and mobile browsers which has scarcely been investigated. Instead of adapting the layout for each device separately, one layout could be optimized to be compatible with different types of online access. As the screens of mobile devices are smaller than computer screens and navigated using fingers instead of the more precise mouse, they represent more restrictions for survey presentation than computers. The most feasible option for a multipurpose design would seem to create one based on this most restricted environment, which is then also applied to the more advanced environments. 
Moreover, it is not known whether the regular web survey layout works inherently better as an input interface than the recently developed layouts for mobile touchscreen interfaces. For example, the relatively large buttons of the touchscreen layout could be an easy-to-use alternative on computer screens as well, especially for those who are less able to pinpoint small radio buttons. Therefore, research is needed to evaluate whether the mobile web layout could be applied to computer web surveys as well. Another important incentive to explore this approach is that the functionality of computers is moving toward touchscreen devices as some laptops are already being equipped with touchscreens.

\section{Methods}

We conducted an experiment in the CentERpanel, a probability sample of households which forms an appropriate estimation of the Dutch-speaking population in the Netherlands. The panel is composed of over 2,000 households which complete web surveys every week. Since not all Dutch people have computers, those households that do not have a computer or Internet access are provided with one to avoid sample bias.

On August 9, 2013, we fielded a questionnaire in the panel to investigate the effects of administering a survey with a mobile web layout. The survey consisted of ten 7-point scale items on social satisfaction, followed by survey evaluation items. All panel members aged 16 and older were invited to participate. Prior to the field work, the sample was split into two random groups. One group was allocated to a regular, computer web version of the questionnaire, with the standard panel layout. Another group was allocated to a survey version which was especially designed for mobile devices and touchscreens. Both questionnaire versions could be opened with regular and mobile web browsers. In Figure 1, examples of both layouts are presented. Using the example of Millar and Dillman (2012), we encouraged respondents using mobile devices in the mobile layout condition to inform the respondents about this possibility and to investigate whether this might stimulate mobile response.

The touchscreen design was developed using the C-MoTo style sheet, a mobile touchscreen interface for Blaise IS questionnaires based on jQuery Mobile. The interface makes use of the most common mobile touchscreen features such as relatively large font size and large buttons for answer options.

The questionnaire consisted of ten items on one's perception of his/her neighborhood. The items had 7-point answer scales which were presented vertically. Only one question was shown per screen. In addition, the respondents were asked to evaluate the user-friendliness of the layout at the end of the questionnaire. This could be reported by four usability items with 7-point scales, measuring completion comfort, ease of use, whether other people would quickly learn to work with the layout, and professional impression. We also registered time stamps and user agent strings. 


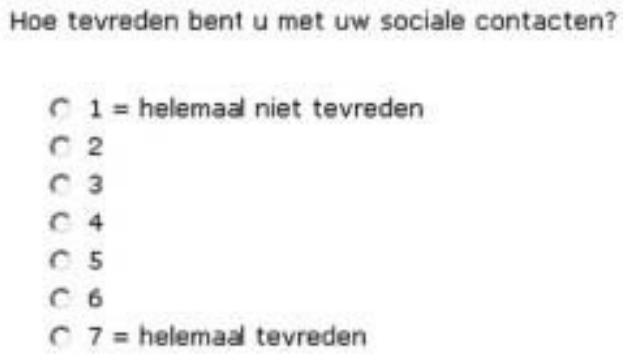

Figure 1 A screenshot of the regular web layout using Chrome.

\begin{tabular}{|l|l|} 
Hoe tevreden bent u met uw sociale contacten? \\
\hline 1 = helemaal niet tevreden \\
\hline 2 \\
\hline 3 \\
\hline 4 \\
\hline 5 \\
\hline 6 \\
$7=$ helemaal tevreden \\
\hline Vorige
\end{tabular}

Figure 2 A screenshot of the mobile web layout using Chrome.

\section{Results}

The response rates were almost identical in both regular (71 percent) and mobile web layout conditions ( 72 percent), and reflected the typical level for the panel (see Table 2). There were virtually no break-off respondents (regular: four respondents and mobile: three respondents). It did take slightly longer for the respondents to complete the mobile web layout version of the questionnaire. 
Table 2 Survey completion by layout.

\begin{tabular}{lll}
\hline & Regular layout & Mobile web layout \\
Number of selected respondents & 1,338 & 1,384 \\
Non-response rate & $29 \%$ & $28 \%$ \\
Completion rate & $71 \%$ & $72 \%$ \\
Median completion time in minutes & 2.9 & $3.1^{\text {a** }}$ \\
\hline
\end{tabular}

${ }^{a}$ Mann-Whitney U test; ${ }^{*} p<0.05 ;{ }^{* *} p<0.01$.

Providing a special design and prompting mobile device usage did not result in more response using smartphones or tablets. In fact, contrary to our expectations, the rate of using mobile devices with the regular web layout was unexpectedly higher (17 percent) than in the mobile web condition (13 percent) $\left[\chi^{2}(1, n=1,961)=4.37, p=0.04\right]$. We checked that there were no significant differences between the conditions by age, sex, or education level.

When comparing survey means by condition, only two out of ten items resulted in significant differences (see Table 3). There were no differences in the frequency of selecting first or last answer options, implying a lack of response order effects by layout. No significant differences in answer distributions could be found on 0.01 level and only one on 0.05 level ( $\chi^{2}$-tests, results not shown). Altogether the different layouts appear not to lead to differences in survey results.

We also compared the results within condition by the device which was used to complete the survey, and between conditions within these device based subgroups. It should be noted that the subgroups were self-selected. We found, however, few significant differences by used device.

Overall, the respondents were less satisfied with the mobile web layout than the regular web layout (see Table 4). When comparing the survey satisfaction between respondents who had used a computer and those who had used a mobile device to complete the survey, the results show that mobile users were clearly more satisfied with the mobile version than the PC users. However, they rated the mobile layout significantly better than the computer layout on only two of four factors, namely the ease of use and learning speed.

When the respondents were asked for suggestions to improve the mobile layout, features such as the color of the survey were often mentioned, as well as the fact that the font was too large or the elements were too widely spread on larger screens. 
Table 3 Survey means and selecting first and last answer options by layout.

\begin{tabular}{|c|c|c|c|c|c|c|}
\hline & \multicolumn{3}{|c|}{ Regular layout } & \multicolumn{3}{|c|}{ Mobile web layout } \\
\hline & \multicolumn{3}{|c|}{ 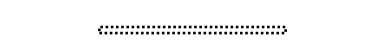 } & \multicolumn{3}{|c|}{ 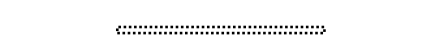 } \\
\hline & $\begin{array}{l}\text { PC } \\
\text { users }\end{array}$ & $\begin{array}{l}\text { Mobile } \\
\text { users }\end{array}$ & Total & $\begin{array}{l}\text { PC } \\
\text { users }\end{array}$ & $\begin{array}{l}\text { Mobile } \\
\text { users }\end{array}$ & Total \\
\hline \multicolumn{7}{|l|}{ Survey means } \\
\hline 1. How satisfied are you with your social contacts? & 5.50 & 5.43 & 5.49 & $5.38^{\mathrm{b} *}$ & 5.41 & 5.39 \\
\hline 2. I have people who I can really talk to. & 5.75 & 5.73 & 5.75 & $5.60^{\mathrm{b*}}$ & 5.67 & $5.61^{\mathrm{b} *}$ \\
\hline 3. I feel isolated from other people. & 2.19 & 2.21 & 2.19 & 2.21 & 2.30 & 2.23 \\
\hline 4. There are people that truly understand me. & 5.25 & 5.44 & 5.28 & 5.16 & 5.25 & 5.18 \\
\hline $\begin{array}{l}\text { 5. I have good contact with my neighbors and other } \\
\text { neighborhood residents. }\end{array}$ & 5.29 & 5.09 & 5.26 & $5.12^{\mathrm{b} *}$ & 4.87 & $5.08^{b * *}$ \\
\hline $\begin{array}{l}\text { 6. People in my neighborhood treat each other } \\
\text { pleasantly. }\end{array}$ & 5.34 & 5.45 & 5.36 & 5.27 & 5.20 & 5.26 \\
\hline 7. I live in a closely-knit neighborhood. & 4.26 & 4.10 & 4.24 & 4.23 & 3.96 & 4.19 \\
\hline $\begin{array}{l}\text { 8. The people in my neighborhood do not all subscribe to } \\
\text { the same values and norms. }\end{array}$ & 3.62 & $3.35^{a *}$ & 3.58 & 3.67 & $3.77^{b *}$ & 3.68 \\
\hline $\begin{array}{l}\text { 9. The people in my neighborhood generally do not get } \\
\text { along well. }\end{array}$ & 2.53 & 2.47 & 2.52 & 2.52 & 2.60 & 2.53 \\
\hline 10. You can trust the people in my neighborhood. & 4.96 & 5.01 & 4.97 & 4.90 & 4.85 & 4.89 \\
\hline \multicolumn{7}{|l|}{ Selecting First and Last Answer Options } \\
\hline Mean \# first options selected/all items & $8 \%$ & $7 \%$ & $7 \%$ & $8 \%$ & $7 \%$ & $8 \%$ \\
\hline Mean \# last options selected/all items & $13 \%$ & $11 \%$ & $13 \%$ & $13 \%$ & $11 \%$ & $12 \%$ \\
\hline
\end{tabular}

${ }_{t}^{\mathrm{a}}$-test for significance within condition, between user groups; ${ }^{*} p<0.05 ;{ }^{* *} p<0.01$.

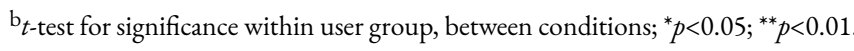

Table 4 Mean scores on survey satisfaction by layout.

\begin{tabular}{|c|c|c|c|c|c|c|}
\hline & \multicolumn{3}{|c|}{ Regular layout } & \multicolumn{3}{|c|}{ Mobile web layout } \\
\hline & \multicolumn{3}{|c|}{ 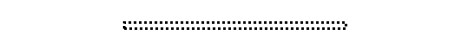 } & \multicolumn{3}{|c|}{ 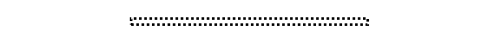 } \\
\hline & $\begin{array}{l}\text { PC } \\
\text { users }\end{array}$ & $\begin{array}{l}\text { Mobile } \\
\text { users }\end{array}$ & Total & $\begin{array}{l}\text { PC } \\
\text { users }\end{array}$ & $\begin{array}{l}\text { Mobile } \\
\text { users }\end{array}$ & Total \\
\hline This layout is pleasant to work with. & 5.75 & 5.58 & 5.72 & $5.17^{\mathrm{b} * *}$ & $5.80^{\mathrm{a} * *}$ & $5.26^{\mathrm{b} * *}$ \\
\hline $\begin{array}{l}\text { It is easy to complete the questions with this } \\
\text { layout. }\end{array}$ & 5.82 & $5.55^{\mathrm{a} *}$ & 5.77 & $5.46^{\mathrm{b} * *}$ & $\begin{array}{l}6.02^{a * *} \\
b * *\end{array}$ & $5.53^{\mathrm{b} * *}$ \\
\hline $\begin{array}{l}\text { People will quickly learn to work with this } \\
\text { layout. }\end{array}$ & 5.56 & 5.48 & 5.55 & $5.37^{\mathrm{b} * *}$ & $5.80^{a * *, b *}$ & $5.42^{\mathrm{b} *}$ \\
\hline This layout has a professional look. & 5.56 & 5.54 & 5.56 & $5.04^{\mathrm{b} * *}$ & $5.61^{a * *}$ & $5.12^{\mathrm{b} * *}$ \\
\hline
\end{tabular}

${ }^{\mathrm{a}} t$-test for significance within condition, between user groups; ${ }^{*} p<0.05 ;{ }^{* *} p<0.01$.

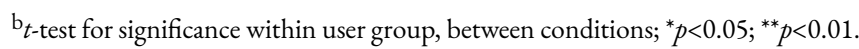




\section{Conclusions}

Web surveys are currently either provided "as is" to all online respondents or, increasingly, adapted for mobile devices. An experiment with an alternative approach, offering a survey with a mobile web layout to the whole web sample, shows that this approach has no effects on the survey outcomes when regular scale items are used.

The layout change on computers does, however, come with the costs of a decrease in survey satisfaction. As Couper (2013) suggests, the fundamental question with mobile data collection is not what is possible, but what the respondents are willing and able to do. There is no technical reason why traditional web survey design cannot adopt features applied in the mobile environment. The main challenges lie in user perceptions. By making survey layout responsive to screen size on computers, font and buttons may become awkwardly large or widely spread on very large screens. Defining a maximum size for the survey window on computers could be one solution to this, but it will take more overall research into the user experience before mobile web survey layout can be applied for computers. Learning from this experiment can nevertheless be a step toward a more advanced survey design in which the best of touchscreen layout is integrated with regular web layout.

\section{Discussion}

While our experiment contained only regular scale items, more research would be needed on comparing the layouts with other question types and different lengths of question text and answer scales. When using large amounts of text or very long answer scales, the limits of visible screen size are reached sooner with the mobile layout than with the traditional web layout.

Finally, when actively designing a multi-device, multi-browser survey, there are two strategic approaches to follow. One can attempt to optimize the survey layout for each type of device separately, or one can pursue a universal, multipurpose survey layout suited for all devices. To arrive at the ideal online survey design requires further research into both strategies. Our experiment already showed that, for regular question items, there should not be a difference in survey results if a mobile layout is used for both mobile and web users, instead of the traditional web layout. This approach brings the practical advantage of being compatible with all most common online devices while requiring less survey development time than multiple survey versions. If the perceived design can be improved such that the respondent satisfaction meets that of the web layout, this approach provides a new strategic choice to seriously consider for online surveys. 


\section{REFERENCES}

Boreham, R., and A. Wijnant. 2013. "Developing a Web-Smartphone-Telephone Questionnaire.” In IBUC 2013 15th International Blaise Users Conference. Washington D.C. http://www.blaiseusers.org/2012/papers/04b.pdf.

Bruijne, M., and A. Wijnant. 2013. "Comparing Survey Results Obtained via Mobile Devices and Computers: An Experiment with a Mobile Web Survey on a Heterogeneous Group of Mobile Devices versus a Computer-Assisted Web Survey.” Social Science Computer Review 31 (4): 482-504.

Buskirk, T.D., and C. Andrus. 2012. "Smart Surveys for Smartphone: Exploring Various Approaches for Conducting Online Mobile Surveys via Smartphones.” Survey Practice. http://surveypractice.wordpress.com/2012/02/21/smart-surveys-for-smart-phones/.

Callegaro, M. 2010. "Do You Know Which Device Your Respondent Has Used to Take Your Online Survey?” Survey Practice. http://surveypractice.wordpress.com/2010/12/08/device-respondenthas-used/.

Couper, M.P. 2013. "Is the Sky Falling? New Technology, Changing Media, and the Future of Surveys." In Keynote Speech Presented at 5th European Survey Research Conference. Ljubljana, Slovenia. July 18, 2013. http://www.europeansurveyresearch.org/conference/couper.

Mavletova, A. 2013. "Data Quality in PC and Mobile Web Surveys.” Social Science Computer Review $31(4): 725-43$.

Millar, M., and D.A. Dillman. 2012. "Encouraging Survey Response via Smartphones.” Survey Practice. https://www.surveypractice.org/article/3095-encouraging-survey-response-viasmartphones.

Stapleton, C.E. 2013. “The Smart(Phone) Way to Collect Survey Data.” Survey Practice. https://www.surveypractice.org/article/3027. 